\title{
LA FORMACIÓN EN SALUD Y SEGURIDAD DEL PROFESORADO PARA LA GESTIÓN DE CENTROS EDUCATIVOS SEGUROS Y SALUDABLES
}

\author{
TEACHERS' TRAINING IN HEALTH AND SAFETY FOR THE MANAGEMENT \\ OF SAFE AND HEALTHY SCHOOLS
}

\author{
Anna Díaz-Vicario1,a (i] , Joaquín Gairín Sallán²,b \\ 1,2 U. Autònoma de Barcelona, España \\ aanna.diaz@uab.cat, bjoaquin.gairin@uab.cat
}

Recibido: 29/07/2021; Aceptado: 06/10/2021

\begin{abstract}
Resumen
Tal y como nos recuerdan la WHO y la UNESCO (2021), la educación y la salud son derechos humanos fundamentales y esenciales que, junto con la necesidad de seguridad y protección, garantizan el desarrollo económico y social. Contar con centros educativos seguros y saludables como espacios donde se promueva y proteja la seguridad y la salud es una cuestión que nadie discute, pues la escuela desempeña un papel fundamental en el desarrollo de las habilidades para la vida. Ahora más que nunca, y tal y como se ha puesto de manifiesto con la situación generada por el virus COVID19, es importante que las escuelas contribuyan al bienestar en un entorno de aprendizaje seguro y saludable para todos los miembros de la comunidad educativa. La creación y gestión de centros seguros y saludables requiere de la puesta en marcha de mecanismos y recursos, así como de la implicación de los equipos directivos y profesorado, sólo posible si cuentan con la formación y herramientas necesarias para ello. La presente aportación analiza, en este contexto, la importancia de la formación del profesorado no universitario, principalmente de educación infantil y primaria, para logar su alfabetización en cuestiones de seguridad y salud escolar, proponiendo un decálogo de competencias e indicadores/estándares de actuación para la gestión de centros seguros y saludables.
\end{abstract}

Palabras clave: Organización escolar; Seguridad; Salud; Formación; Alfabetización.

\begin{abstract}
As the WHO and UNESCO (2021) remind us, education and health are fundamental and essential human rights that, together with the need for security and protection, guarantee economic and social development. The foundation of safe and healthy educational institutions, as spaces where safe and health are promoted and protected, is an issue that no one disputes since schools play a fundamental role in developing life skills. Now more than ever, and as the situation generated by the COVID19 virus has shown, schools must contribute to well-being in a safe and healthy learning environment for all members of the educational community. Create and manage safe and healthy schools requires developing mechanisms and resources and involve management teams and teachers. Those are only possible if all the actors involved have the necessary training and tools to do so. This contribution analyses, in this context, the importance of train non-university teachers, mainly of childhood and primary education, to achieve their health and safety literacy, proposing a decalogue of competencies and indicators/standards of action for the management of safe and healthy schools.
\end{abstract}


Keywords: School organization; Safety; Health; Training; Literacy.

\section{INTRODUCCIÓN}

Los centros educativos deben ser entornos escolares seguros y saludables (también sostenibles, aunque no sea objeto de esta aportación) en donde profesorado, alumnado y el resto de los miembros de la comunidad educativa gocen de bienestar físico, emocional y social y ausencia de riesgos para su salud y seguridad. La situación provocada por la COVID-19 ha subrayado más esta necesidad, planteando que estas instituciones necesariamente deben ser espacios protegidos donde se preserve el logro de los resultados educativos. Pero, más allá de las circunstancias actuales provocadas por la pandemia que nos afecta, la promoción y el desarrollo de escuelas seguras y promotoras de salud no es algo nuevo, pues su necesidad fue planteada hace ya más de 25 años, aunque hasta el momento ha tenido un desarrollo bastante desigual entre los países europeos y casi inexistente en el caso español (Ayuso et al., 2018).

En un momento en el que las escuelas se consideran cada vez más entornos clave para promover la salud, el bienestar y el desarrollo de niños y jóvenes (WHO y UNESCO, 2021) y para educar en la salud, seguridad, prevención y protección (Instituto Nacional de Seguridad y Salud en el Trabajo [INSST], 2018), es necesario que los centros educativos adopten un enfoque integral sobre la promoción de la seguridad y salud escolares. Deben atender las condiciones físicas, socioemocionales y psicológicas del entorno escolar, así como alfabetizar el alumnado en salud y seguridad, convirtiendo a la escuela en un ejemplo de entorno seguro y saludable. Y para que ello ocurra, los profesionales de la educación han de contar con las competencias necesarias (Burgos-García, 2019; Cajina-Pérez, 2020; Kingston et al., 2018), aunque, actualmente, la formación recibida sigue siendo más bien escasa (Charro-Huerga y Charro, 2017; Llorent-Bedmar y Cobano-Delgado, 2019; Torres-García y Santana-Hernández, 2017).

Asegurar la formación y capacitación del profesorado para la educación y promoción de la seguridad y la salud en el entorno escolar es una actuación clave. Al respecto, la presente aportación persigue un doble objetivo: a) Caracterizar los centros educativos seguros y saludables que promueven y educan en esa perspectiva; y b) Reflexionar sobre los conocimientos, capacidades, actitudes, valores y creencias con los que han de contar el profesorado para gestionar centros educativos seguros y saludables.

Inicialmente, clarificamos la relación entre los conceptos de seguridad, salud y educación, para posteriormente adentrarnos en la definición y caracterización de los centros educativos como organizaciones seguras y saludables. Delimitado el marco de actuación, se aborda la importancia de la formación de los maestros en materia de seguridad y salud escolar, concretando cuáles serían las competencias clave a desarrollar en la formación de maestros y aportando algunas claves para su desarrollo. La perspectiva adoptada se enmarca en los Objetivos de Desarrollo Sostenible (ODS) aprobados en 2015 por la Asamblea General de las Naciones, en la línea de garantizar una vida sana y una educación de calidad contando con centros educativos seguros y saludables donde se promueve el bienestar de todos sus integrantes.

\section{SEGURIDAD, SALUD Y EDUCACIÓN}

Seguridad, salud y educación son tres conceptos inseparables. Para garantizar procesos de aprendizaje efectivos es necesario contar con ambientes seguros y saludables, pues un alumno sano, que se siente seguro en la escuela, aprende mejor, al igual que enseña mejor un maestro 
que goza de buena salud y se siente seguro (Díaz-Vicario, 2017). Estudios previos han demostrado que una mejor salud y bienestar de los estudiantes, incluyendo el comportamiento saludable y el desarrollo de habilidades socioemocionales, están asociados con una mayor asistencia a la escuela, motivación, participación y rendimiento académico (Basch, 2011; Durlak et al., 2011; Martínez-Vicente et al., 2019; Owen et al., 2018). Pero ¿qué entendemos por seguridad y salud y cómo se vinculan ambos términos con la educación?

La Organización Mundial de la Salud [OMS] define la salud como "un estado de completo bienestar físico, mental y social, y no solamente la ausencia de afecciones o enfermedades" (Constitución de la Organización Mundial de la Salud, 1948, preámbulo). Una escuela que promueve la salud es "una escuela que se caracteriza por fortalecer constantemente su capacidad como espacio saludable para vivir, aprender y trabajar" (OMS, 2020). Dado que nuestro bienestar está condicionado por factores ambientales, crear entornos saludables, entre los que se incluye la escuela, es imprescindible para garantizar una buena salud en la infancia y la juventud (Llorent-Bedmar y Cobano-Delgado, 2019). Por ello, se habla de crear entornos saludables, como espacios donde se promueve un ambiente sano en el que aprender de modo saludable y solidario con el medio (Gavidia, 2001), partiendo de una concepción holística y ecológica de la salud que considere las dimensiones física, emocional y social (Chamarro et al., 2009).

Por su parte, la seguridad es definida desde múltiples perspectivas. En un sentido amplio, la seguridad es entendida como la cualidad de aquello que se encuentra "libre y exento de todo peligro, daño o riesgo" (Real Academia Española, 2020). Concretamente, una escuela segura es conceptualizada como una escuela "que no es peligrosa y que no plantea amenazas a los ocupantes de la escuela en términos de bienestar físico, emocional y psicológico" (Xaba, 2014, p. 1583). La Iniciativa Mundial para Escuelas Seguras de la Alianza Global para la Reducción del Riesgo de Desastres y Resiliencia en el Sector Educación (2018) señala que las escuelas seguras cuentan con instalaciones de aprendizaje seguras y accesos y egresos seguros, está preparada para dar respuesta a emergencias en la escuela y proporciona educación para la reducción del riesgo de desastres y resiliencia.

Si unimos los conceptos de seguridad y salud en educación, de lo que se trata es que las escuelas sean un espacio seguro donde se proteja la salud física, emocional y social y se promueva el bienestar de todos los integrantes, a la vez que se potencia la formación en estos ámbitos. Así adoptamos el término escuelas seguras y saludables, en el sentido de que se trata de emplear medidas apropiadas para asegurar que los centros educativos sean espacios seguros y saludables para todos aquellos que los utilizan, apoyando el crecimiento académico, social y emocional de los estudiantes (Voon y Mohammad, 2019).

Las concepciones más avanzadas de seguridad superan la mera preocupación por la seguridad física (condiciones adecuadas del entorno formativo: climatización, sonido, luz, etc.) y hablan también de la seguridad desde el punto de vista emocional y social (Díaz-Vicario, 2015). Se introduce así una perspectiva dinámica (ver Figura 1) que considera aspectos que enlazan con las dimensiones personales y sociales de la salud y que justifica ampliamente el que relacionemos los conceptos de seguridad y salud y de que hablemos de seguridad integral y de salud integrada con otros aspectos de la realidad personal, institucional y social y que afecta por igual a estudiantes, profesorado y otros miembros de la comunidad educativa. 
Figura 1. Referentes de la seguridad integral: dimensión estática y dinámica del riesgo (Equipo de Desarrollo Organizacional [EDO], 2013).

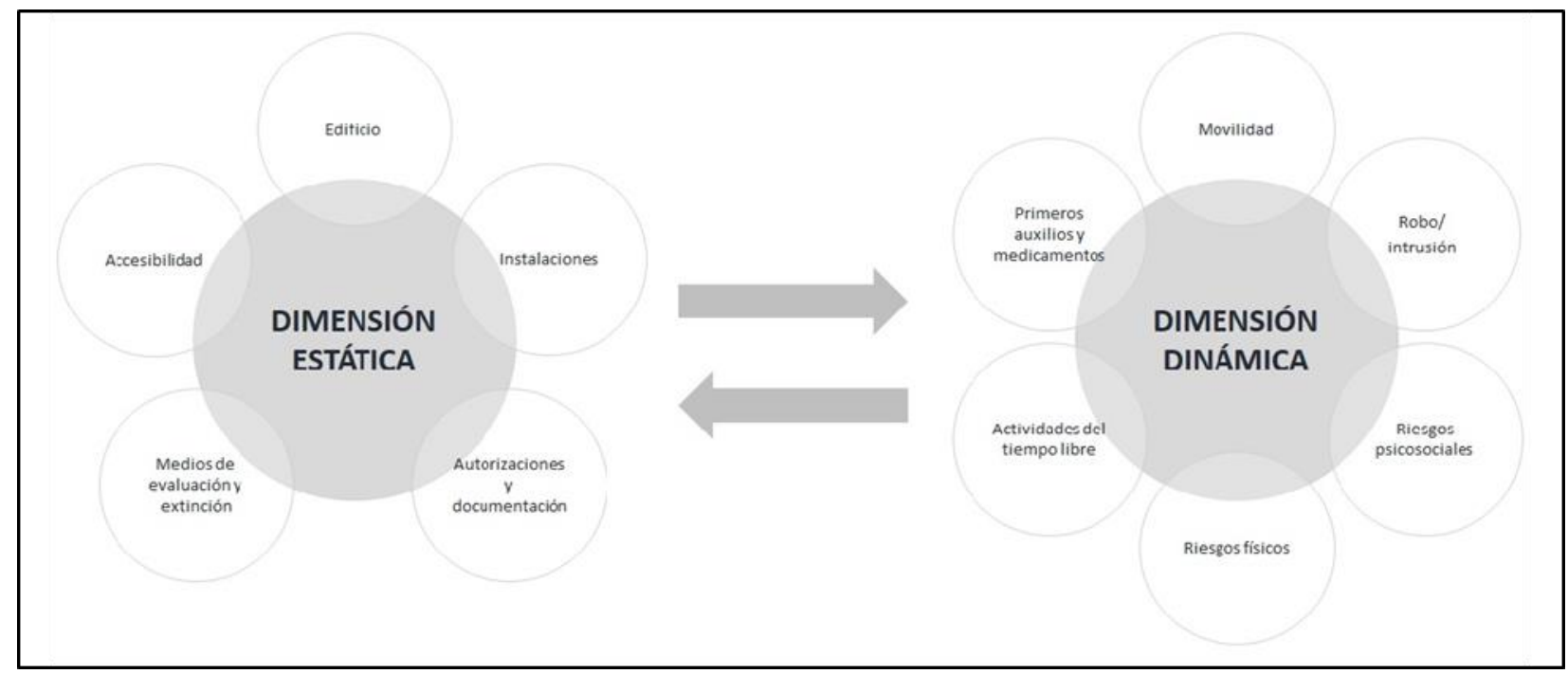

\section{CENTROS EDUCATIVOS SEGUROS Y SALUDABLES}

La necesidad de promover escuelas seguras y saludables fue introducida hace ya más de 25 años (WHO y UNESCO, 2021) ${ }^{1}$. Desde entonces, algunos progresos se han realizado a través de iniciativas como la Red de Escuelas Promotoras de Salud (en inglés, Schools for Health [SHE]) o la Iniciativa Mundial para Escuelas Seguras (WISS, por sus siglas en inglés), aunque de un modo desigual en los diferentes países europeos. Asimismo, se han desarrollado diversos modelos, que, generalmente, ponen el foco en los estudiantes, cuando en el día a día de los centros conviven tanto estudiantes como personal docente y no docente, sin contar otros miembros de la comunidad educativa (padres, asesores del centro, visitantes, etc.) que también interactúan con ellos.

Igualmente, desde 2017, el Ministerio de Educación está impulsando los Planes estratégicos de convivencia escolar y de hábitos saludables (Rosales, 2020), buscando crear marcos de colaboración institucional entre administraciones y agentes implicados en la promoción de estilo de vida saludables, el diseño de estrategias para la promoción de la salud en el ámbito escolar, y el fomento de la formación e investigación al respecto, así como el desarrollo de una Red Estatal de Escuelas Promotoras de Salud (MECD, 2017).

El contexto escolar es una comunidad en la que conviven alumnos, personal docente y no docente, familias y otros especialistas (psicopedagogos, profesores específicos, personal vinculado a los servicios del centro, monitores, etc.), compartiendo un espacio común. Fomentar y conseguir un ambiente de estudio sano y seguro exige adoptar un enfoque comprensivo que una las perspectivas educativa, organizacional, cultural y de diseño (Duke 2002). De acuerdo con Longás et al. (2014), tan importante será atender los retos y problemas de la seguridad y salud comunes a diversos colectivos, a través de la gestión de recursos compartidos, como aquellas cuestiones de carácter más específico que afectan particularmente a cada colectivo.

Un centro educativo seguro y saludable es aquel que gestiona los recursos estructurales y organizativos para garantizar el bienestar físico, emocional y social de todos los miembros de la comunidad educativa, velando por la creación de entornos seguros y saludables (Díaz-Vicario y Gairín, 2020). Hablamos de un entorno ejemplar en cuanto a la gestión de la salud y la 
seguridad se refiere, en donde no sólo se vigilen las instalaciones y equipos escolares, sino también los procedimientos, las metas, la cultura y el clima que se promueve.

El Modelo de la organización escolar segura y saludable de Díaz-Vicario y Gairín (2020) distingue tres dimensiones interdependientes (ver Figura 2) que los centros educativos deben atender:

- Ambiente físico y estructural. Incluye elementos vinculados al edificio escolar, accesos, espacios de juego y equipamientos del recinto escolar y alrededores, considerando también la distribución de las aulas y otros espacios docentes, así como las condiciones higiénico-sanitarias.

- Ambiente psicosocial. Integra los elementos que inciden en la organización del trabajo (modelo organizativo, clima de centro, relaciones internas y externas, etc.) y en la cultura organizacional (valores, creencias, actitudes y actuaciones cotidianas), que afectan a la salud y al bienestar físico y socioemocional de alumnos y personal docente y no docente.

- Prácticas organizativas. Contempla los procesos organizativos establecidos y que abarcan tanto la concreción y realización de planes y programas de intervención como la actuación de los agentes (equipos directivos y otro personal) en su ejecución y coordinación, incluyendo también los necesarios sistemas de comunicación.

El modelo está en línea con modelos comprensivos y holísticos que enfatizan que promover la seguridad y la salud escolar incluye múltiples factores y actividades que afectan tanto al entorno escolar, como al currículum y a la comunidad (Lee et al., 2020). Asimismo, entiende, desde una perspectiva dinámica, que las tres dimensiones se vinculan entre sí y lo hacen proactivamente y a partir de los procesos organizativos dirigidos a prevenir, intervenir, proteger, e incluso actuar en situaciones de emergencia. De nuevo volvemos a considerar el marco escolar como un entorno sistémico con componentes variados y como espacio de síntesis de influencias internas y externas. 
Figura 2. Modelo de la organización escolar segura y saludable (Gairín y Díaz-Vicario, 2019, p. 3).

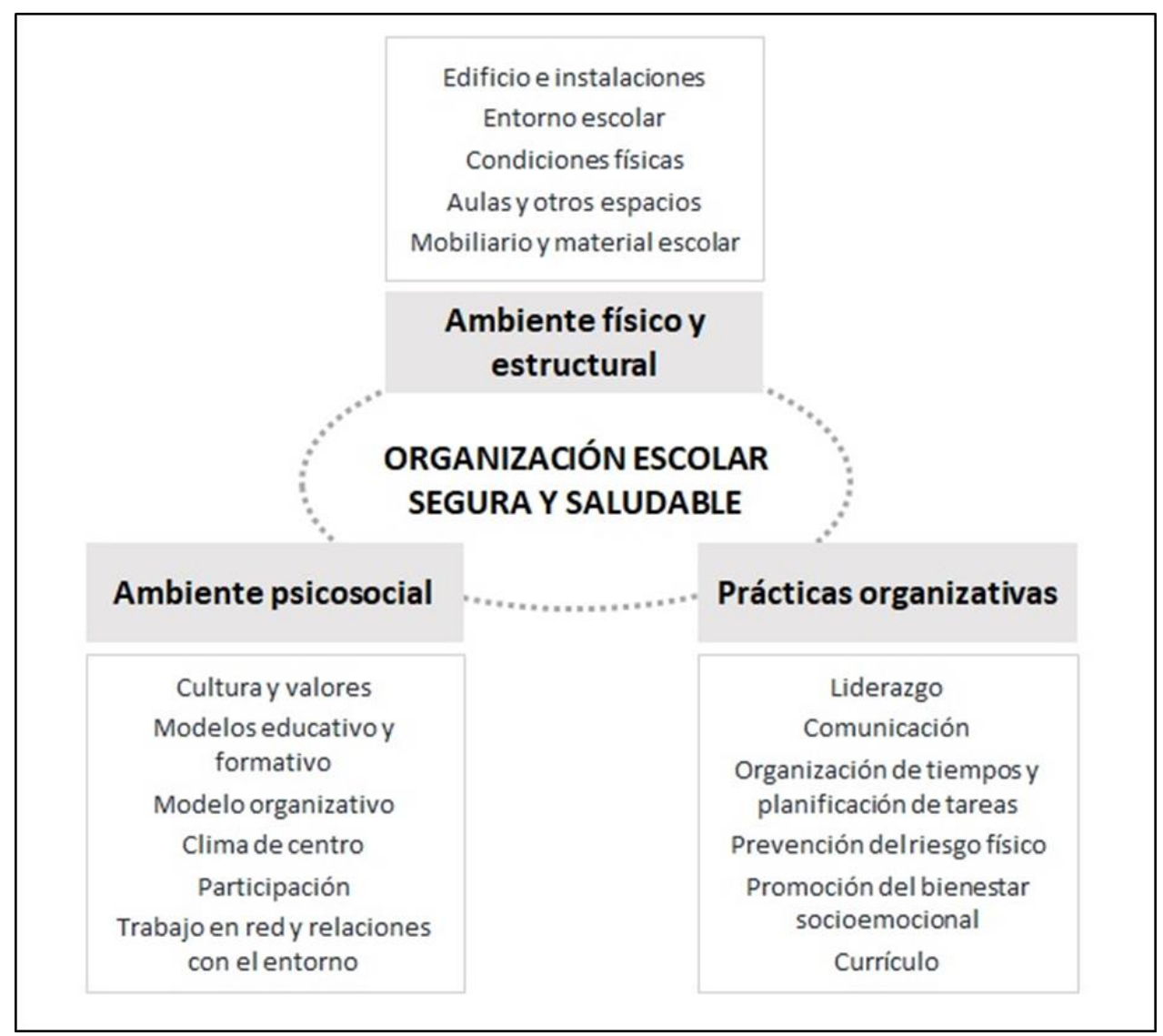

De este modo, un centro educativo seguro y saludable se caracteriza por integrar la visión de la seguridad y la salud escolar en todas y cada una de las actividades que desarrolla como institución, empezando por reflejarse en su Proyecto Educativo. De un modo más específico, es un centro que:

a. Cuenta con un ambiente físico de la escuela (incluyendo tanto el edificio escolar como el entorno escolar próximo) diseñado para promover la seguridad (mínimo riesgo para los usuarios), la salud y el bienestar de los miembros de la comunidad educativa.

b. Atiende adecuadamente las relaciones interpersonales y la resolución de conflictos, para garantizar un adecuado clima de estudio y trabajo, entendiendo que unas malas relaciones alteran no sólo la realidad emocional de las personas sino y también su capacidad para disfrutar y aprender de las relaciones con otras personas.

c. Desarrolla programas y actuaciones que previenen el riesgo y promueven el bienestar de todos los miembros de la comunidad educativa.

d. Educa en hábitos y actitudes que fomentan conductas seguras y saludables.

Conseguir los propósitos que se mencionan es una tarea colectiva que implica a todos los miembros de la comunidad educativa, que se comprometen y participan activamente en la creación y mantenimiento de un centro escolar seguro y saludable.

\section{FORMACIÓN DEL PROFESORADO EN SEGURIDAD Y SALUD ESCOLAR}

Promover un centro seguro y saludable implica necesariamente contar, en primer lugar, con profesorado sensibilizado y formado. De hecho, tal y como ya hemos apuntado previamente, 
uno de los principales obstáculos para integrar escolarmente la educación en seguridad y salud, es la escasa formación con la que cuentan los maestros, aun y cuando la importancia de la formación en estas áreas se ha abordado desde diferentes organizaciones e instituciones (Burgos-García, 2019).

Actualmente, la asignatura de educación para la salud, en la mayoría de los casos, es una materia optativa o inexistente en los planes de estudio del Grado de Maestro en las universidades españolas (Charro-Huerga y Charro, 2017) y lo mismo sucede en el caso de la formación en materia de seguridad y prevención (Burgos-García, 2013). No es de extrañar, por tanto, que los estudiantes universitarios de magisterio tengan escasos conocimientos tanto en educación para la salud (Cubero et al., 2011), como en seguridad escolar, aun cuando existen experiencias aisladas que, como las conducidas por Torres-García y Santana-Hernández (2017), indican que incluir una asignatura de formación básica en el plan de estudios contribuye a una mejor percepción de los conocimientos y capacidades necesarias para desarrollar estrategias de promoción y educación para la salud en los centros educativos. En cualquier caso, son diversas las voces que abogan por fortalecer tanto los conocimientos teóricos como los metodológicos y de concienciación en seguridad y salud escolar (Ayuso et al., 2018).

Tomando como referencia las definiciones de alfabetización para la salud de la WHO y la UNESCO (2021), podemos decir que la formación en seguridad y salud del profesorado está vinculada a la alfabetización funcional de este colectivo. Supone que dispongan del conocimiento, la motivación y las aptitudes para acceder, comprender y aplicar la información en temas de seguridad y salud, para prevenir situaciones que la pongan en riesgo, y para fomentar acciones y estrategias que ayuden a mantener o mejorar el bienestar físico, emocional y social tanto desde un punto de vista individual como colectivo.

\section{Competencias y estándares de actuación del profesorado alfabetizado en salud y seguridad escolar}

La gestión de la seguridad y la salud en los centros educativos, así como la formación de los maestros para impartir contenidos vinculados con educación en seguridad y salud, requiere contar con docentes capacitados en la temática, más cuando la educación para la salud es un principio pedagógico recogido en la propia legislación educativa de muchos países.

Hayes (2003), quien analizó las áreas de competencia docente, destaca explícitamente la responsabilidad del profesional de la educación para garantizar la seguridad, la salud y el bienestar del alumnado. De hecho, si analizamos las funciones del profesorado recogidas en la legislación vigente (artículo 91 de la LOE que sigue vigente con la nueva Ley orgánica (LOMLOE) recientemente aprobada (2020) y que modifica los artículos a y g), observamos que, más allá de las funciones propias relacionadas con el proceso de enseñanza-aprendizaje, la tutoría, la orientación y el acompañamiento y la organización y gestión del centro, se hace referencia a los aspectos psicosociales, ámbito que debe impulsarse para crear un centro seguro y saludable. Específicamente, los artículos mencionados hacen referencia a:

e) La atención al desarrollo intelectual, afectivo, psicomotriz, social y moral del alumnado.

g) La contribución a que las actividades del centro se desarrollen en un clima de respeto, de tolerancia, de participación y de libertad para fomentar en los alumnos los valores de la ciudadanía democrática y de la cultura de paz.

Hayes (2003) identifica 7 competencias clave y 2 estándares de actuación del profesorado. Si los analizamos, en dos de las siete competencias incorpora descriptores que se vinculan con la creación de entornos seguros y saludables para el aprendizaje. Igualmente, en ambos 
estándares de actuación se identifican hasta siete descriptores que enfatizan en valores y prácticas profesionales, así como en el proceso de enseñanza en sí mismo y que subrayan el papel clave de los maestros para garantizar la seguridad y salud física, social y emocional y el bienestar de los estudiantes en el entorno escolar (ver Tabla 1).

Tabla 1. Identificación de competencias y estándares de actuación de los maestros vinculados con la promoción de la salud, la seguridad y el bienestar del alumnado. Díaz-Vicario (2015, p. 189), a partir de Hayes (2003).

\begin{tabular}{|c|c|c|}
\hline & & SELECCIÓN DE ENUNCIADOS \\
\hline \multirow{3}{*}{ 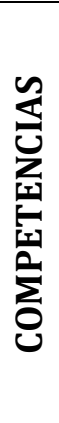 } & \multirow{2}{*}{$\begin{array}{l}\text { Enfoque } \\
\text { pedagógico }\end{array}$} & $\begin{array}{l}\text { - Establecer y mantener un ambiente de trabajo seguro y un } \\
\text { ambiente disciplinado. }\end{array}$ \\
\hline & & $\begin{array}{l}\text { Establecer un entorno seguro que apoye el aprendizaje y en el que } \\
\text { los alumnos se sientan seguros y confiados. }\end{array}$ \\
\hline & $\begin{array}{l}\text { Métodos y } \\
\text { estrategias de } \\
\text { enseñanza- } \\
\text { aprendizaje }\end{array}$ & $\begin{array}{l}\text { - Aprovechar las oportunidades para mejorar las habilidades básicas } \\
\text { de los alumnos, señalando que los recursos deben estar disponibles } \\
\text { y ser accesibles, y garantizando, también, que sean seguros y que se } \\
\text { encuentren en buen estado. }\end{array}$ \\
\hline \multirow{7}{*}{ 窟 } & & $\begin{array}{l}\text { - Tratar a los alumnos con respeto y consideración y mostrar } \\
\text { preocupación por su desarrollo. }\end{array}$ \\
\hline & $\begin{array}{l}\text { Valores y } \\
\text { prácticas } \\
\text { profesionales }\end{array}$ & $\begin{array}{l}\text { - Ser consciente del marco legal de responsabilidad de los maestros, } \\
\text { hecho que implica: (a) ser consciente de las cuestiones de } \\
\text { protección de la infancia; (b) estar familiarizado con los } \\
\text { procedimientos de actuación en el caso del alumnado con NEE; (c) } \\
\text { adoptar una actitud responsable hacia la seguridad de los niños en } \\
\text { el centro educativo y durante las visitas; y (d) ser consciente de la } \\
\text { necesidad de mantener un enfoque profesional en el centro } \\
\text { educativo. }\end{array}$ \\
\hline & \multirow{5}{*}{ Enseñanza } & $\begin{array}{l}\text { - Utilizar estándares objetivos de enseñanza-aprendizaje para } \\
\text { planificar las lecciones, mostrando cómo van a evaluar a los } \\
\text { alumnos, garantizando que el bienestar emocional de todos los } \\
\text { alumnos sea respetado y atendido. }\end{array}$ \\
\hline & & $\begin{array}{l}\text { - Seleccionar y preparar recursos, así como planificar su } \\
\text { organización segura y eficaz, considerando los intereses, lengua y } \\
\text { cultura de los estudiantes, hecho que comporta atender las } \\
\text { cuestiones de salud y seguridad en el despliegue y uso de los } \\
\text { equipos. }\end{array}$ \\
\hline & & $\begin{array}{l}\text { - Planificar actividades fuera del contexto del centro educativo para } \\
\text { ofrecer otras oportunidades de aprendizaje al alumnado, hecho que } \\
\text { comporta tomar conciencia acerca de los factores de seguridad y } \\
\text { salud y sus implicaciones. }\end{array}$ \\
\hline & & $\begin{array}{l}\text { - Tener altas expectativas de los estudiantes, construir relaciones } \\
\text { exitosas centradas en la enseñanza-aprendizaje y establecer un } \\
\text { ambiente de aprendizaje en el que la diversidad sea valorada y los } \\
\text { alumnos se sientan seguros y confiados. }\end{array}$ \\
\hline & & $\begin{array}{l}\text { Organizar y gestionar el espacio físico de enseñanza, así como las } \\
\text { herramientas, materiales, textos y otros recursos de manera segura } \\
\text { y efectiva. }\end{array}$ \\
\hline
\end{tabular}


Este mismo autor, destaca algunas de las actuaciones que evidenciarían que los docentes dominan las competencias relativas a la seguridad y la salud (Hayes, 2003, p. 131, citado en Díaz-Vicario, 2015, p. 189-190) y que sintetizamos a continuación:

- Sensibilidad hacia los potenciales peligros físicos y la vulnerabilidad de los alumnos, a través de la realización de evaluaciones de riesgos antes de llevar a cabo las actividades, orientación clara y sin ambigüedades sobre los factores y los procedimientos de seguridad, y atención a los niños especialmente vulnerables.

- Planificación y evaluación de riesgos para evitar la exposición a los peligros, garantizando la idoneidad y seguridad de las actividades y vigilando los movimientos del alumnado. Ello supone evaluar el riesgo de cada actividad sobre la base de las directrices de seguridad y salud establecidas por el centro educativo, contando con el asesoramiento de docentes experimentados.

- Comportamiento responsable: mostrar entusiasmo hacia el trabajo, escuchar a los demás, hacer todo lo posible por cumplir con las prioridades del centro educativo y no comprometer la propia integridad ni la de los demás.

Enlazando con la propuesta anterior, Fortuny (1992) considera que la actuación del profesorado debe ir dirigida a cuatro direcciones:

a. El medio material, vigilando que el edificio, las instalaciones, los equipamientos, los materiales y los recursos cumplan las normativas de seguridad.

b. El medio psicosocial, creando un ambiente escolar democrático, abierto y afectivo que favorezca la comunicación, el diálogo, el reconocimiento y el bienestar emocional.

c. La vigilancia permanente del estudiantado, extremando dicha vigilancia en las horas de entrada y salida, en los recreos, en las salidas escolares y en las clases de educación física, laboratorio y talleres.

d. Las conductas del profesorado, siendo coherentes con sus comportamientos personales y los comportamientos que intentan favorecer en sus estudiantes.

Si nos centramos en los contenidos, Burgos-García (2019) nos señala que la formación en seguridad y salud de los maestros debería integrar tanto conocimientos legislativos, como técnicos y científicos-académicos, además de comportamientos y actitudes. Por ello destaca que los contenidos y/o campos de acción que los alumnos de las facultades de educación deberían aprender se relacionan con (Burgos-García y Llacuna, 2011, p. 2):

a. Edificio y equipo: análisis del aula y recursos materiales con los que se cuenta, ya sea otorgados por la administración educativa y laboral como las que puedan ser diseñadas 'in situ'.

b. Organización y desarrollo de la organización: analizar el clima, normativa interna de organización y funcionamiento o el proyecto educativo del centro, elementos que intervienen en el proceso comunicativo y participación.

c. Enseñanza de la prevención: contenido de los temas, metodología y evaluación de la propuesta preventiva a enseñar, capacidad para el trabajo en equipo, capacidad reflexiva, etc.

d. Condiciones políticas y marco social: regulaciones y leyes, plan de estudios y pautas, trabajar con los padres y las tecnologías al servicio de la enseñanza y la prevención. 
Los principales objetivos de cualquier acción formativa de los maestros deben ser: a) concienciar de que la seguridad y la salud son parte integral de cualquier actividad educativa; b) sensibilizar sobre la necesidad de crear entornos escolares seguros y saludables que velen por el bienestar de estudiantes y maestros, c) familiarizarlos con los aspectos básicos vinculados con la gestión y la organización escolar que contribuyen a la seguridad y la salud, y e) inculcar la importancia y necesidad de plantear los aspectos vinculados con la seguridad y la salud como un elemento de enseñanza para generar en los estudiantes comportamientos y actitudes acordes.

Teniendo en cuenta lo anterior, y partiendo del concepto de centro educativo como espacio seguro y saludable expuesto anteriormente, así como de las referencias previas, hacemos una propuesta de decálogo sobre las competencias e indicadores/estándares de actuación que, desde la visión del Modelo de organización escolar segura y saludable, debería tener un profesor/a formado en la materia.

\section{Competencias del profesorado para la gestión de centros escolares seguros y saludables}

Las competencias clave que un profesor/a debe poseer para impulsar la gestión de centros escolares seguros y saludables son:

1. Actuar con responsabilidad y de acuerdo con los principios deontológicos de la profesión docente, evitando poner en riesgo la propia seguridad y salud, así como la de los estudiantes a su cargo y otros miembros de la comunidad educativa.

2. Generar propuestas que promuevan la seguridad, la salud y el bienestar de los miembros de la comunidad educativa.

3. Aplicar los fundamentos de la perspectiva de los centros escolares seguros y saludables.

4. Crear y mantener un entorno escolar seguro y saludable que apoye el proceso de aprendizaje del alumnado.

5. Diseñar espacios de aprendizaje que garanticen la seguridad y el bienestar de estudiantes y maestros.

6. Examinar los determinantes sociales, los riesgos para la salud y los factores de protección que afectan a los estudiantes.

7. Reordenar las situaciones que pueden poner en riesgo la seguridad, la salud y el bienestar de los miembros de la comunidad educativa.

8. Participar en la promoción de hábitos y comportamientos seguros y saludables entre los estudiantes.

9. Participar, junto con el resto de los miembros de la comunidad educativa, en la creación y mantenimiento de una cultura de seguridad y salud en el centro educativo.

10. Responsabilizarse de la propia seguridad y salud en el entorno escolar.

\section{Indicadores/estándares de actuación del profesorado alfabetizado funcionalmente en seguridad y salud escolar}

El desarrollo de las competencias señaladas con los procesos y metodologías adecuadas nos ha de permitir verificar el grado de consecución de las conductas deseables que, en nuestro caso, se pueden concretar en indicadores que bien podrían transformarse en estándares si se delimita el sistema de medición. Serían los siguientes: 
1. Muestra una actitud e implicación coherente con los principios de la promoción de la seguridad y la salud en el entorno escolar.

2. Participa en el establecimiento y desarrollo de políticas escolares que promuevan la seguridad, la salud y el bienestar de la comunidad educativa.

3. Vela para que los fundamentos de la perspectiva de los centros escolares seguros y saludables estén presentes en cualquier decisión adoptada individual 0 colectivamente.

4. Crea y mantiene un entorno escolar seguro y saludable que apoye el proceso de aprendizaje de los estudiantes.

5. Organiza y gestiona el espacio de aprendizaje de forma segura para promover la salud y el bienestar.

6. Promueve un ambiente respetuoso basado en relaciones positivas como base para la creación de un entorno escolar que promueve el bienestar de la comunidad educativa.

7. Reporta a la dirección del centro o autoridades competentes los riesgos identificados y que pueden afectar negativamente la seguridad y salud de la comunidad educativa, participando en la búsqueda de soluciones conjuntas.

8. Proporciona a los estudiantes conocimientos y habilidades indispensables para adoptar decisiones responsables respecto a su salud y bienestar personal, así como para el desarrollo de la salud y seguridad de su entorno.

9. Se implica en la creación y mantenimiento de una cultura de seguridad y salud en el centro educativo.

10. Identifica y utiliza los recursos existentes en su entorno para proteger su propia seguridad y salud.

En definitiva, asumir la necesidad de entornos seguros y saludables y actuar proactivamente en conseguirlos, en el marco de una cultura institucional que hace de ello un propósito institucional.

\section{Otras cuestiones a considerar}

Delimitadas las competencias e indicadores que se podrían relacionar con el logro de estas, su consecución y aplicación efectiva se vincula a otros temas contextuales y organizativos que habría que considerar y que se comentan a continuación.

Consideradas la seguridad y la salud como un tema transversal (Rosales, 2020, así lo considera), su ubicación en el currículo universitario podría dar lugar a una materia específica o bien incluir sus propósitos y contenidos en las materias que hablan del contexto institucional. De todas formas, su trabajo sistemático a través de una materia o parte de otra no puede obviar su consideración en otras materias, sobre todo las didácticas, a la hora de organizar los procesos de enseñanza-aprendizaje.

Tan importante como los objetivos didácticos, derivados de las competencias y considerando los niveles de logro que se desean, es la determinación de la metodología de trabajo. En nuestro caso, y considerando que es importante incidir en las actitudes y comportamientos, será importante utilizar el método de casos y partir de ejemplos reales próximos o lejanos, promoviendo la expresión de las opiniones y conductas. En la misma dirección, cabrá considerar una evaluación de logros a partir de proyectos o tomando como referencia asignaturas transversales como puede ser el prácticum. 
Garantizar una buena formación en los temas que tratamos exige que el profesorado universitario implicado conozca las temáticas y los formatos cómo se trabajan en los centros educativos. Al respecto, y para garantizarla, parece conveniente pensar en actividades formativas dirigidas a este profesorado, como en su momento se hizo cuando se trató de impulsar los modelos inclusivos o ahora cuando se habla de competencia digital docente.

La propuesta formativa creemos que no es suficiente para conseguir las transformaciones efectivas que se desean, si no va acompañada de un contexto sensibilizado y practicante de las propuestas de entornos seguros y saludables. Además de formar en esas temáticas, las instituciones formadoras deben de presentarse también como seguras y saludables, participando también de redes, talleres, campañas de sensibilización, formación e investigación sobre ellas. Recordamos, en este contexto, que las organizaciones y contextos, como los profesionales, también pueden educar a través de las actuaciones que hacen o promueven.

En todo caso, el programa formativo sobre centros educativos seguros y saludables ha de estar integrado en el plan curricular de la formación inicial del profesorado, utilizar metodologías recurrentes y plantearse desde perspectivas colaborativas para que pueda incidir en el cambio de actitudes y no sólo de conocimientos.

\section{A MODO DE CONCLUSIÓN}

Organizaciones internacionales, como la OMS y la OSHA, reivindican la promoción de la salud en los centros escolares y la formación en una cultura de seguridad y salud, argumentando que desde la educación se puede lograr la alfabetización en seguridad y salud de las futuras generaciones. Es necesario contar, al respecto, con ambientes escolares que promuevan la seguridad y la salud y también con profesionales de la educación formados para ello. Más allá de los contenidos que puedan integrarse en los currículums de infantil, primaria y secundaria, se trata de alinear el diseño del entorno escolar con los principios de seguridad y salud para que sea un ejemplo tanto de un espacio seguro y saludable como de comportamientos acordes a este principio (Voon y Mohammad, 2019).

Pero crear entornos educativos seguros y saludables requiere de una adecuada organización y gestión del centro educativo en dicha materia y eso sólo será posible si contamos con profesorado formado en la materia. Deben tener un conocimiento que les permita tomar decisiones e intervenir en la gestión del centro y del aula, siendo necesario un planteamiento formativo que forme en conocimientos, además de en comportamientos y actitudes.

La propuesta de formación presentada parte de las consideraciones realizadas tanto por organismos internacionales (ENETOSH, SHE, WHO, UNESO) como por expertos en el ámbito de la educación para la salud y la seguridad (Burgos-García y Llacuna, 2011; Hayes, 2003). Sin la pretensión de exponer una propuesta cerrada, hemos esbozado las competencias y los indicadores/estándares de actuación de un profesor alfabetizado funcionalmente en materia de seguridad y salud a partir de los cuales podrían derivarse los contenidos a desarrollar a través de acciones de formación vinculadas a la formación inicial y continua de los profesionales de la educación.

\section{REFERENCIAS BIBLIOGRÁFICAS}

Alianza Global para la Reducción del Riesgo de Desastres y Resiliencia en el Sector Educación (2018). Iniciativa Mundial para Escuelas Seguras. "En el 2030 toda escuela será segura". Recuperado de https://www.unicef.org/lac/informes/iniciativa-mundial-para-escuelas-seguras 
Ayuso, R., Medina, J. L., y Molina, M. C. (2018). Educación para la salud en la escuela. Análisis reflexivo a través de la legislación educativa. Campo Abierto, 37(2), 203-216. https://mascvuex.unex.es/revistas/index.php/campoabierto/article/view/3260

Basch, C. E. (2011). Healthier students are better learners: a missing link in school reforms to close the achievement gap. The Journal of school health, 81(10), 593-598. https://doi.org/10.1111/i.1746-1561.2011.00632.x

Burgos-García, A. (2013). Prevención de riesgos laborales en los centros educativos: Análisis de los sistemas de formación. Revista de Educación, (361), 37-64. https://www.educacionyfp.gob.es/dam/jcr:79750783-f239-420e-bddfee1adc0a3bef/re36102-pdf.pdf

Burgos-García, A. (2019). Formación para la salud y seguridad escolar. European Journal of Health Research, 5(1), 17-30. https://doi.org/10.30552/ejhr.v5i1.134

Burgos-García, A. y Llacuna, J. (2011). La formación inicial universitaria de maestros/as de educación infantil y primaria en PRL. Notas técnicas de prevención, 920. Instituto Nacional de Seguridad e Higiene en el Trabajo. https://www.insst.es/documents/94886/328579/920w.pdf/af5b4967-d7fa-4c66-9e18b3af7fbebcdb

Cajina-Pérez, L. (2020). Importancia de la Educación para la Salud en currículo educativo. Revista Electrónica de Conocimientos, Saberes y Prácticas, 3(1), 170-180. https://doi.org/10.5377/recsp.v3i1.9799

Constitución de la Organización Mundial de la Salud. Conferencia Sanitaria Internacional, Nueva York, 19/06-22/7/1946, firmada el 22/07/1946 por los representantes de 61 Estados, y que entró en vigor el $07 / 04 / 1948$.

Cubero, J., Calderón, M., Costillo, E., y Ruiz, C. (2011). La educación para la salud en el Espacio Europeo de Educación Superior. Publicaciones, (41), 51-63. https://doi.org/10.5209/RCED.51536

Chamarro, A., Longás, E., Longás, J., y Capell, M. (2009). Danys no intencionals a l'escola. Gestió de la seva prevenció. SAIP-Blanquerna Assistencial i de Serveis (Universitat Ramón Llull).

Charro-Huerga, E. y Charro, M. E. (2017). Formación del profesor de primaria en educación para la salud. Didáctica de las ciencias experimentales y sociales, 32(1), 183-201. https://doi.org/10.7203/dces.32.9968

Díaz-Vicario, A. (2015). La gestión de la seguridad integral en los centros educativos: facilitadores y obstaculizadores [Tesis de Doctorado, Universitat Autònoma de Barcelona]. Repositorio - TESEO

Díaz-Vicario, A. (2017). Escuelas seguras y saludables. Guía para la Gestión de Centros Educativos, PD52017, 1-19. Wolters Kluwer España

Díaz-Vicario, A., y Gairín, J. (2020). Organizaciones escolares seguras y saludables. Revista MC Salud Laboral, febrero, 10-13. Recuperado de https://www.mcmutual.com/documents/20143/2872819/organizaciones escolares.pdf/4ba421d0-d3bb4531-4615-9ddb9bd95108

Duke, D. L. (2002). Creating safe schools for all children. Allyn \& Bacon.

Durlak, J. A., Weissberg, R. P., Dymnicki, A. B., Taylor, R. D., y Schellinger, K. B. (2011). The impact of enhancing students' social and emotional learning: a meta-analysis of school-based universal interventions. Child development, 82(1), 405-432. https://doi.org/10.1111/j.14678624.2010.01564.x

Equipo de Desarrollo Organizacional [EDO]. (2013). Edurisc. Recuperado de http://edo.uab.cat/edurisc Fortuny, M. (1992). Educació per a la seguretat i prevenció d'accidents a les institucions educatives. Temps d'Educació, (8), https://raco.cat/index.php/TempsEducacio/article/view/140344

217-228.

Gairín, J. y Díaz-Vicario, A. (2019). Los centros educativos como organizaciones seguras y saludables. Resultados de un estudio diagnóstico. DYLE - Revista de Dirección y Liderazgo Educativo, (3), 110. Recuperado de https://www.dyle.es/los-centros-educativos-como-organizacionesseguras-y-saludables-resultados-de-un-estudio-diagnostico/

Gavidia, V. (2001). La transversalidad y la escuela promotora de salud. Revista Española de Salud Pública, 75(6), 505-516. http://dx.doi.org/10.15658/INVESTIGIUMIRE.201101.06

Hayes, D. (2003, 2 ${ }^{\mathrm{a}}$ ed.). Planning, teaching and class management in primary schools. Routledge. 
Instituto Nacional de Seguridad y Salud en el Trabajo (INSST) (2018). La seguridad y la salud como materia de enseñanza en la educación primaria. Guía para el profesorado. Instituto Nacional de Seguridad y Salud en el Trabajo (INSST). Recuperado de https://bit.ly/3hRXf36

Kingston, B., Mattson, S. A., Dymnicki, A., Spier, E., Fitzgerald, M., Shipman, K., Goodrum, S., Woodward, W., Witt, J., Hill, K. G., y Elliott, D. (2018). Building Schools' Readiness to Implement a Comprehensive Approach to School Safety. Clinical child and family psychology review, 21(4), 433-449. https://doi.org/10.1007/s10567-018-0264-7

Lee, A., Lo, A., Li, Q., Keung, V., y Kwong, A. (2020). Health Promoting Schools: An Update. Applied Health Economics and Health Policy, 18(5), 605-623. https://doi.org/10.1007/s40258-020-00575-8

Longás, E., Longás, J., y Riera, J. (2014). Daños no intencionados en la escuela. Estudio de su incidencia en escolares de Cataluña. Educar, 50(2), 243-263. https://doi.org/10.5565/rev/educar.42

Llorent-Bedmar, V. y Cobano-Delgado, V. (2019). La formación en educación para la salud del alumnado universitario del grado de educación infantil en España. Ciência \& Saúde Colectiva, 24(8), 30673078. https://doi.org/10.1590/1413-81232018248.28642017

Ley Orgánica 2/2006, de 3 de mayo, de Educación. Boletín Oficial del Estado, núm. 106, de 04 de mayo de 2006. Referencia: BOE-A-2006-7899.

Ley Orgánica 8/2013, de 9 de diciembre, para la mejora de la calidad educativa. Boletín Oficial del Estado, núm. 295, de 10 de diciembre de 2013. Referencia: BOE-A-2013-12886.

Martínez-Vicente, M., Suárez-Riveiro, J.M., y Valiente-Barroso, C. (2019). Estrés cotidiano infantil y factores ligados al aprendizaje escolar como predictores del rendimiento académico. Ansiedady Estrés, 25(2), 111-117. https://doi.org/10.1016/j.anyes.2019.08.002

MECD (2017). Planes estratégicos de convivencia escolar y hábitos saludables. Recuperado de https://sede.educacion.gob.es/publiventa/plan-estrategico-de-salud-escolar-y-estilos-de-vidasaludable-2016-2020/educacion-salud/22124

OMS (1986). Carta de Ottawa para la Promoción de la Salud. Conferencia Internacional sobre la promoción de la salud. OMS/Salud y Bienestar social Canadá/ Asociación canadiense de salud pública.

OMS (2020). What is a health promoting school? [Entrada web]. Recuperado de https://www.who.int/publications/i/item/9789240025059

Owen, K. B., Parker, P. D., Astell-Burt, T., y Lonsdale, C. (2018). Regular Physical Activity and Educational Outcomes in Youth: A Longitudinal Study. The Journal of adolescent health: official publication of the Society for Adolescent Medicine, 62(3), 334-340. https://doi.org/10.1016/j.jadohealth.2017.09.014

Real Academia Española (2020). Seguridad En Diccionario panhispánico del español jurídico. Recuperado de https://dpej.rae.es/lema/seguridad

Rosales, C. (2020). Escenarios y estrategias para el aprendizaje transversal. Innovación educativa, (30), 57-73. https://doi.org/10.15304/ie.30.7011

Torres-García, M. y Santana-Hernández, H. (2017). La Educación para la Salud en la formación de maestros desde el Espacio Europeo de Educación Superior. Revista Complutense de Educación, 28(4), 1083-1101. https://doi.org/10.5209/RCED.51536

Voon, H. y Mohammad, T. (2019). Safety and Health in Schools. International Journal of Recent $\begin{array}{llll}\text { Technology and Engineering (IJRTE), 251-258. } & \text { 8(2S3), }\end{array}$ https://doi.org/10.35940/ijrte.B1043.0782S319

WHO y UNESCO (2021). Making every school a health-promoting school. Global standards and indicators. WHO and UNESCO. Recuperado de https://www.who.int/news-room/articles-detail/globalstandards-and-indicators-for-health-promoting-schools-and-their-implementation-guidance

Xaba, M. I. (2014). A Holistic Approach to Safety and Security at Schools in South Africa. Mediterranean Journal of Social Sciences, 5(20), 1580-1589. https://doi.org/10.5901/mjss.2014.v5n20p1580 


\section{NOTAS}

${ }^{1}$ Como antecedente significativo se puede citar la Carta de Ottawa de la OMS (1986), dirigida a la consecución del objetivo 'Salud para todos en el año 2000', y donde se menciona el rol de las escuelas, como los hogares y lugares de trabajo, en la creación de hábitos y en la preparación de las personas para afrontar las enfermedades y lesiones crónicas. 